Gestión de la seguridad y salud del trabajo desde la perspectiva de la cognición

\title{
Gestión de la seguridad y salud del trabajo desde la perspectiva de la cognición
}

\author{
Gestión de la seguridad y salud del trabajo desde la \\ perspectiva de la cognición
}

Gestão de segurança e saúde ocupacional na perspectiva da cognição

Pavel Omar Defranc Balanzategui I
paveldefranc@hotmail.com
Roberto Bolívar Oviedo Quiñónez II
robertoviedo_q@ hotmail.com
Jhonny Xavier Tomalá Sánchez
jhonnyxavier1976@hotmail.com

Recibido: 30 de enero de $2017 *$ Corregid: 20 de febrero de $2017 *$ Aceptado: 20 junio de 2017

I. Magister de Seguridad, Higiene Industrial y salud Ocupacional Universidad de Guayaquil. Docente y Tutor en Tesis de Maestrías, Guayaquil, Ecuador.

II. Magister de Seguridad, Higiene Industrial y salud Ocupacional Universidad de Guayaquil. Docente y Tutor en Tesis de Maestrías, Docente y Tutor de Posgrado Decanato de Ingeniería Industrial, Guayaquil, Ecuador.

III. Magister en Seguridad, Higiene Industrial y Salud Ocupacional Universidad de Guayaquil. Capacitador y Asesor en Seguridad y Salud Ocupacional, Guayaquil, Ecuador. 


\section{Resumen}

La investigación se realizó con el propósito de evaluar las condiciones y medio ambiente de trabajo en función de los sistemas de gestión de la seguridad y salud del trabajo en varias empresas de la provincia de Guayas, Ecuador, en aras de fortalecer las debilidades incorporando estrategias conducentes a un mejoramiento continuo de las prácticas de trabajo. El diseño de la investigación estrategica fue de campo, permitiendo la evaluación del trabajador en la ejecución de las prácticas de trabajo en cuanto a las normas enfocadas a la seguridad y salud laboral, así como, la actuación cognitiva en el hacer de las tareas.Y según el propósito de la investigación es proyectiva porque se propone un modelo de gestión de seguridad. Además de estrategias hacia la ejecución de mejoras continuas. Para establecer las estrategias se utilizó la matriz de fortalezas, debilidades, oportunidades y amenazas. Se definieron estrategias como; estandarización de programas, creacción de grupos interactivos en los diferentes niveles de trabajo, aplicación de asesorias externas multidisciplinaria, estandarización de métodos de reclutamiento y selección del personal, implementación de planes de monitoreo, instituir alianzas con los trabajadores y otras empresas, implantar mecanismos de auditorias, entre otros. Se precisó que los sistemas de gestión deben trabajar de forma integral para lograr fortalecer las debilidades existentes en la toma de decisiones encausadas hacia la calidad de la gestión de seguridad y salud del trabajo.

Palabras claves: Gestión de la seguridad, salud, prácticas de trabajo, cognitiva, mejora continua, estrategias. 


\begin{abstract}
The research was carried out with the purpose of evaluating the working conditions and environment in function of occupational safety and health management systems in several companies in the province of Guayas, Ecuador, in order to strengthen the weaknesses by incorporating leading strategies to a continuous improvement of work practices. The design of the strategic research was of field, allowing the evaluation of the worker in the execution of the work practices regarding the norms focused on occupational health and safety, as well as, the cognitive performance in the doing of the tasks. According to the purpose of the research, it is projective because a security management model is proposed. In addition to strategies towards the execution of continuous improvements. To establish the strategies, the matrix of strengths, weaknesses, opportunities and threats was used. Strategies were defined as; program standardization, creation of interactive groups at different levels of work, application of multidisciplinary external consultancies, standardization of recruitment methods and selection of personnel, implementation of monitoring plans, instituting alliances with workers and other companies, implementation of audit mechanisms , among others. It was specified that the management systems must work in an integral way to strengthen the existing weaknesses in the decision-making process aimed at the quality of occupational health and safety management.
\end{abstract}

Key words: Management of safety, health, work practices, cognitive, continuous improvement, strategies. 


\section{Introducción.}

La evolución del hombre ha generado cambios importantes en el desarrollo tecnológico e innovador de los países. Ha contribuido con los avances productivos de las empresas y con ello ha favorecido a la sociedad incorporando beneficios que esten más alla de una simple producción.

En tal sentido, desde la edad antigua el ser humano se ve en la necesidad de crear mecanismos conducentes hacia la salud del trabajador. Sin embargo, con el avance del tiempo y por evaluaciones realizadas por investigadores como Frederick Taylor, determinaron, que no todo queda en la parte de la salud por lo que surge la seguridad del trabajo.

González expresa que la seguridad en el trabajo es la disciplina teórico-práctica que se sirve de un conjunto de técnicas y procedimientos que tienen por objeto eliminar o reducir el riesgo de que se produzcan accidentes de trabajo (2003, p. 8). Mientras que, Chinchilla Sibaja, (2002)expone que es la encargada de atender una serie de peligros que inciden en los accidentes laborales.

Ahora bien, la Salud del trabajo está orientada hacia el cuidado del trabajador de manera que se garantice el estado mental, físico y social en condiciones adecuadas.

Las empresas, con respecto a la seguridad y salud en el trabajo han incorporado mecanismos que le permitan minimizar los riesgos y eliminar los accidentes en su totalidad en función de las actividades que intervienen en sus procesos productivos. Sin embargo, año tras año, siempre han tenido resultados significativos en cuanto a los incidentes y accidentes ocurridos en sus empresas.

La Organización Internacional del Trabajo (OIT) en el Congreso Mundial Sobre Seguridad y Salud en el Trabajo celebrado en el año 2014 hace referencia que "2,3 millones de personas mueren cada año alrededor del mundo a causa de accidentes y enfermedades relacionadas con el trabajo". 
También expresa que, "cada día ocurren 860.000 accidentes en el trabajo con consecuencias en términos de lesiones". Situación que genera costos directo e indirectos de los accidentes y enfermedades profesionales, los cuales se estima en 2,8 billones (millones de millones) de dólares.

Por lo planteado el director general de la OIT expresó que esas cifras son inaceptables, situación que lleva a reflexión porque los accidentes laborales graves son en primer lugar tragedias humanas, pero la economía y la sociedad también pagan un precio alto.

En Ecuador por un artículo desarrollado en El Telégrafo, 2012 se detecta un número significativo de accidentes de trabajo reportados. Es decir, para el año 2011 se registraron 9305 y para el año 2012 hasta el mes de septiembre ya se reportaban 7677. Datos suministrados por el Seguro General deRiesgo del Trabajo del Instituto Ecuatoriano de Seguridad Social.

Por otro lado Sotomayor, 2016 desarrollo un artículo donde señala que desde el año 2010 en Ecuador se viene presentando un incremento exponencial de algún tipo de accidente laboral. Manifiesta que por investigaciones realizadas, la Universidad Internacional SEK, en 2010, indico que aproximadamente 7904 personas sufrieron accidente, incrementandose hacia el 2015 en 24379. Seguido hacia el año 2016 con 62500 trabajadores.

Después de las consideraciones anteriores, y así como otros países, Ecuador ha procurado fortlecer sus normativas con la intención de optimizar la gestión estratégica y operativa de las empresas. En consecuencia promueven la Constitución de la República del Ecuador, 2008 la cual considera diferentes garantías hacia los derechos y deberes. Tal es el caso del artículo 326, número 5, que toda persona tendrá derecho a desarrollar sus labores en un ambiente adecuado y propicio, que garantice su salud, integridad, seguridad, higiene y bienestar. 
Sin embargo, a pesar de los esfuerzos realizados aún se presenta en el tiempo un porcentaje sustancial de accidentes que conduce a cuestionarse sobre la forma de llevar las actividades enmarcadas en la Seguridad y Salud del Trabajo.

Por lo planteado se pregunta ¿Cuál es la perspectiva cognitiva del trabajador hacia la seguridad y salud del mismo?. La cognición, esta relacionada con el saber del ser humano y la conducta que presenta ante las actividades. Entonces, ¿Por qué si es capacitado, no actua adecuadamente ante los eventos inesperados?¿Será qué el hombre solo adquirió conciencia colectiva de proteccion, pero no individual?. Por otro lado, se cuestiona ¿Cómo controlo o minimizo las enfermedades o accidentes de las empresas?

Es de mencionar que inicialmente se crean manuales de seguridad industrial y con ello se inicia la aplicación de los mismos en sus procesos. Sin embargo, las empresas se han dado cuenta que no solamente la seguridad debe ir encaminada con base a procedimientos establecidos en un manual, sino que debería ser considerada como una gestión.

La gestión de la seguridad y salud ocupacional es uno de los objetivos que más le preocupa y ocupa a las empresas debido a los altos porcentajes de los índices de accidentabilidad que año tras años se vienen presentando. En consecuencia ha ido creando nuevas estrategias que permitan minimizar los riesgos y los posibles accidentes laborales.

Sobre la base de las consideraciones anteriores, se planteó una investigación de tipo proyectiva debido a que surge la necesidad de evaluar y proponer estrategias que conduzcan a optimizar los Sistemas de Gestión de la Seguridad y Salud del Trabajo en las empresas. 


\section{Materiales y Métodos.}

Para el desarrollo de la investigación se hizo necesario realizar investigaciones en las empresas con el propósito de evaluar el comportamiento de los trabajadores hacia la ejecución de las prácticas del trabajo, haciendo énfasis en los sistemas de gestión de la seguridad y salud laboral.

En tal sentido según la estrategia la investigación realizada fue de campo. De acuerdo con Hernández, (2006), se entiende por Investigación de Campo:

Análisis sistemático de problemas en la realidad, con el propósito bien sea de describirlos, interpretarlos, entender su naturaleza y factores constituyentes, explicar sus causas y efectos, o predecir su ocurrencia, haciendo usos de métodos característicos de cualquiera de los paradigmas o enfoque de investigación conocidos o en desarrollo. Los datos de interés son recogidos en forma directa de la realidad; en este sentido se trata de investigaciones a partir de datos originales o primarios (Pág. $18)$.

Por otro lado según el propósito es proyectiva porque se propone un modelo de gestión de seguridad y salud del trabajo que establece la relación empresa-trabajador desde la incorporación del ciclo de Deming el cual se basa en planificar, hacer, verificar y actuar (PHVA), y la fomentación del mejoramiento continuo hacia la obtención de buenas prácticas de seguridad y salud del trabajo.

Hurtado de Barrera, (2010)señala que la investigación proyectiva propone soluciones a partir de un proceso de indagación, que conlleva a proponer alternativas de cambios. 
Para la articulación de la información se considero la aplicación de la matriz de evaluación fortalezas, debilidades, oportunidades y amenzas (FODA) con el propósito de establecer las estrategias conducentes a las mejoras de los sistemas de gestión de seguridad y salud del trabajo.

(Fernandez, 2003)expresa que la matriz FODA es una herramienta de ajuste importante que ayuda a crear cuatro tipos de estrategias; estrategias de fortalezas y oportunidades (FO), estrategias de debilidades y oportunidades (DO), estrategias de fortalezas y amenazas (FA) y estrategias de debilidades y amenazas (DA).

\section{Resultados.}

Herbert William Heinrich fue el primer ingeniero de seguridad reconocido y quien realizó miles de estudios y análisis de accidentes donde expresó que de cada 75,000 accidentes, 10\% se debe a condiciones peligrosas, $88 \%$ a condiciones inseguras y $2 \%$ a actos imprevistos. Concluyendo que de cada 100 accidentes 98 pudieron ser previstos.

Las normas ISO, s.f. ha desarrollado pautas con el propósito de incorporar medidas que permitan optimizar la gestión empresarial. Las ISO 45001, Sistemas de Gestión de Salud y Seguridad Ocupacional es una de las normas que ayudará a las organizaciones en función de la seguridad de sus empleados y los riesgos en los sitios de trabajo de manera que se pueda crear condiciones y medio ambiente de trabajo mejores y con seguridad.

Es de señalar que la ISO 45001 surge inicialmente porque de acuerdo a las investigaciones de la Organización Internacional de Normalización detectaron que más de 6300 personas mueren cada día de accidentes o enfermedades relacionadas con el trabajo, eso es casi 2.3 millones cada año. 
Con referencia a lo anterior surge la aprobación de la ISO 45001 como un proyecto de la Norma Internacional, así lo manifiesta Gasoriowski-Denis, (2016) expresando que el mundo está a un paso hacia las mejoras de la seguridad en el trabajo en las cadenas de suministros globales, lo que conllevaría a minimizar las lesiones en el lugar de trabajo y las enfermedades en el mundo.

Ahora bien, el Instituto Ecuatoriano de Seguridad Social (IESS) es una entidad que se encarga de aplicar el sistema del seguro social obligatorio que forma parte del sistema nacional de Seguridad Social. Con base a lo planteado es que (ALTAÏR, 2017) enuncia que las empresas deben tener e implantar un Sistema de Gestión en Seguridad y Salud Laboral que además de permitirles estar ajustados a las normativas les garantizará el éxito en la disminución de los accidentes de trabajo y de las enfermedades profesionales.

Dadas las condiciones que preceden, se realizaron estudios a diferentes empresas en aras de identificar las actitudes del trabajador cuando realiza sus actividades y se relaciona con el medio ambiente de trabajo. Resultando lo siguiente:

- Manipulación inadecuada de herramientas, equipos, y materiales.

- Generación de desperdicios en los alrededor de los puestos de trabajo.

- Posturas forzadas.

- Uso inadecuado de los equipos de proteccion personal.

- Poca importancia hacia el uso de los equipos de protección personal.

- Perturbaciones hacia otros trabajadores, como juegos, bromas, entre otros, al momento de realizar las tareas.

- Irresponsabilidad afectando su seguridad y la de otros trabajadores.

- Tensión por exceso de trabajo y por presencia doble.

- Fatigas por las cargas de trabajo o por el tiempo de la jornada laboral.

- Exceso de confianza al realizar las prácticas de trabajo. 
Por lo planteado, se evidencia que aunque existe charlas, inducciones, y otros tipos de capacitaciones que van a fortalecer tanto el desarrollo del trabajador como sus conocimientos hacia las labores que ejecuta y el medio ambiente de trabajo en el que éste se desenvuelve, el mismo no presenta compromiso.

Sobre la base de las consideraciones anteriores, las empresas en función de buscar alternativas que den respuestas más precisas, se ven en la necesidad de evaluarse, con el propósito de identificar cuáles son las fortalezas y debilidades que presentan a nivel de su gestión en la seguridad y salud del trabajo, así como detectar que oportunidades puede estar desaprovechando hacia su crecimiento competitivo, condición que le podría afectar en su efectividad y eficiencia empresarial. En consecuencia, a continuación se presenta el cuadro 1 donde se señala tanto los factores internos como externos hacia la gestión de la seguridad y salud de trabajo.

Cuadro 1. Matriz FODA

\begin{tabular}{|l|l|}
\hline \multicolumn{2}{|c|}{ Factores Internos } \\
\hline Pertalezas & \multicolumn{1}{|c|}{ Debilidades } \\
\hline $\begin{array}{l}\text { Disposición de los equipos de protección } \\
\text { de seguridad }\end{array}$ & Compromiso \\
\hline Respaldo gubernamental & Actitud hacia el uso de los equipos \\
\hline Disposición de normas & Cumplimiento de las normas \\
\hline Mecanismos de capacitación & Disposición del personal hacia su seguridad \\
\hline Programa de seguridad y salud del trabajo & Desactualización de los programas \\
\hline Disposición de técnicas metodológicas & Indicadores de medición \\
\hline Demarcación de áreas y puestos de trabajo & Condiciones de espacios desordenados \\
\hline & Incidentes y accidentes laborales \\
\hline
\end{tabular}


Gestión de la seguridad y salud del trabajo desde la perspectiva de la cognición

\begin{tabular}{|l|l|}
\hline \multicolumn{2}{|c|}{ Factores Externos } \\
\hline Oportunidades & \multicolumn{1}{|c|}{ Amenazas } \\
\hline Certificación & Acción legal \\
\hline Nuevos mercados & Acción gubernamental \\
\hline Captación de personal calificado & Desincorporación de la empresa \\
\hline Respuestas oportunas & Sindicatos \\
\hline Ambientes de trabajos más seguros & \\
\hline Alianzas estratégicas con empresas & \\
\hline Mediciones de control & \\
\hline
\end{tabular}

Fuente: Elaboración Propia

Con base en la evaluación presentada en la matriz FODA se hace necesario ajustar las fortalezas, debilidades, oportunidades y amenazas en función de establecer estrategias que conduzcan a optimizar las desviaciones presentadas, logrando así una minimización de riesgos y eliminación de accidentes.

En relación con la matriz FODA es importante señalar que esta técnica permite elaborar estrategias en función de cuatro enfoques:

- Estrategias FO. Estrategias en función de la relación fortalezas y oportunidades de manera tal, que la empresa pueda consolidarse y crecer en el tiempo.

- Estrategias DO. Estrategias enfocada a superar las debilidades y aprovechar las oportunidades.

- Estrategias FA. Estrategias conducentes hacia el aprovechamiento de las fortalezas para evitar o disminuir las repercusiones de las amenazas.

- Estrategias DA. Estrategias defensivas que pretenden reducir las debilidades internas y evitar las amenazas del entorno. 
Por el desarrollo de la matriz FODA se pudo realizar evaluaciones que permitieron generar estrategias hacia fortalecimiento y la mejora de la gestión de seguridad y salud del trabajo. Las estrategias determinadas serán definidas a continuación.

\section{Estrategias FO.}

1. Estandarizar los programas de seguridad y salud del trabajo considerando las normas nacionales e internacionales.

Es decir los programas deben estar ajustados a normativas que les permitan medirse y compararse con otras empresas, así podría establecer acciones de mejoras encauzadas a buenas prácticas independientemente de la razón social de la misma.

2. Constituir grupos interactivos en los diferentes niveles organizacionales con el propósito de tener las condiciones y medio ambiente de trabajos seguro.

Con la participación de un personal proactivo y dispuesto a asumir retos alineados al crecimiento de la empresa se pueden fomentar acciones de mejora hacia un ambiente de trabajo adecuado y seguro.

\section{Implementar técnicas metodológicas y mecánismos de capacitación que} garanticen respuestas oportunas del personal para cualquier situación.

Consiste en detallar con exactitud los procedimientos y las condiciones laborales a fin de capacitar al trabajador adecuadamente y con ello pueda responder con efectividad ante cualquier información o evento presentado. 


\section{Estrategias DO.}

1. Emplear asesorias externas multidisciplinarias para la generación y consolidación de los programas.

Las asesorias externas permitirán establecer procedimientos y acciones acordes a los cambios normativos y gubernamentales. Acciones que además de fortalecer los programas, también podrían incidir en la actitud del trabajador hacia la seguridad de sus compañeros y de él mismo.

2. Fortalecer las politicas de la seguridad y salud del trabajador por medio de seminarios y talleres prácticos.

La relación activa del trabajador con entes externos además de permitirles fortalecer sus conocimientos, podría influir en su comportamiento lo cual conllevaría a tener ambientes de trabajos limpios y seguros, mimimizando los incidentes y accidentes.

3. Estandarizar un método de reclutamiento y selección del personal que garantice trabajadores comprometido hacia la seguridad y salud laboral.

Incorporar trabajadores comprometidos hacia la seguridad y salud laboral fomenta compromiso, disposición y cambios culturales que disminuirán las debilidades.

\section{Estrategias FA.}

1. Elaborar planes que permitan monitorear $y$ detectar los cambios gubernamentales y las reformulaciones de las normativas legales que incidan en la gestión de la seguridad y salud del trabajo. 
Revisar y detectar cambios oportunamente permitirá mantener actualizados los programas, por lo cual se minimizan las amenazas externas y se garantiza la calidad hacia le gestión estratégica de la empresa.

\section{Establecer alianzas con los trabajadores para el desarrollo de la gestión} empresarial.

Crear planes a corto plazo que permitan la integración del personal en la gestión con el propósito de influir en su comportamiento y con ello en la operatividad efectiva de los procesos.

\section{Implantar mecanismos de auditoria que permitan detectar las condiciones de} riesgo oportunamente.

Realizar auditorias internas al menos dos veces al año. Acción que, además de permitir detectar las fallas y los riesgos, conducirá a mantener las condiciones y medio ambiente de trabajo acordes a los procesos, elevará la calidad de vida del trabajador minimizando las afecciones que pudieran incidir en su salud y contribuirá en la calidad de la gestión.

\section{Estrategias DA.}

1. Establecer alianzas con otras empresas en aras de fortalecer los niveles de competitividad a través del mejoramiento continuo.

Establecer acuerdos y relaciones con otras empresas en corto y mediano plazo, podría inducir a estar ajustados a las normas y medidas de control. También impulsaría el desarrollo y capacitación del trabajador. 


\section{Implantar planes que involucren al trabajador en el desarrollo de la gestión y en}

\section{los beneficios económicos que obtenga la empresa.}

Alinear al trabajador hacia la misión y visión de la empresa es fundamental para su crecimiento. Pero aún más, es cuando lo incorporas a las acciones de mejora y de decisión porque adquiere sentido de pertenencia. Condición que lo lleva a cambios culturales y comportamientos equilibrados hacia la operacionalización exitosa de la gestión estratégica y gerencial de la empresa.

3. Implantar indicadores de medición para evaluar y detectar oportunamente las condiciones de riesgos que pudieran repercutir en algún incidente o accidente.

Los indicadores de medición permitirán ver el comportamiento cuantitativo y asociarlo a las evaluaciones de manera que se pueda realizar acciones de mejora en tiempo oportuno y así evitar resultados que conlleven a penalizaciones legales.

La aplicación conjunta de las estrategias determinadas permitiran fortalecer la gestión de seguridad y salud del trabajo, porque precisa de acciones que van más alla de un mejoramiento continuo.

Además de la incorporación y aplicación de las estrategias definidas, se debe hacer mucho énfasis en la inclusión y participación del trabajador desde que inicia en la empresa,en función del desarrollo de las actividades y responsabilidades de las mismas, independientemente del puesto de trabajo que ocupa.

En definitiva lo anterior conduce a optimizar la gestión de seguridad y salud del trabajador. A continuación se presenta la figura 1, donde se representa esquematicamente un modelo de gestion de seguridad y salud del trabajo que integra a la empresa, al trabajador y a la sociedad. 


\section{Figura 1. Modelo de Gestión de la Seguridad y Salud del Trabajo}

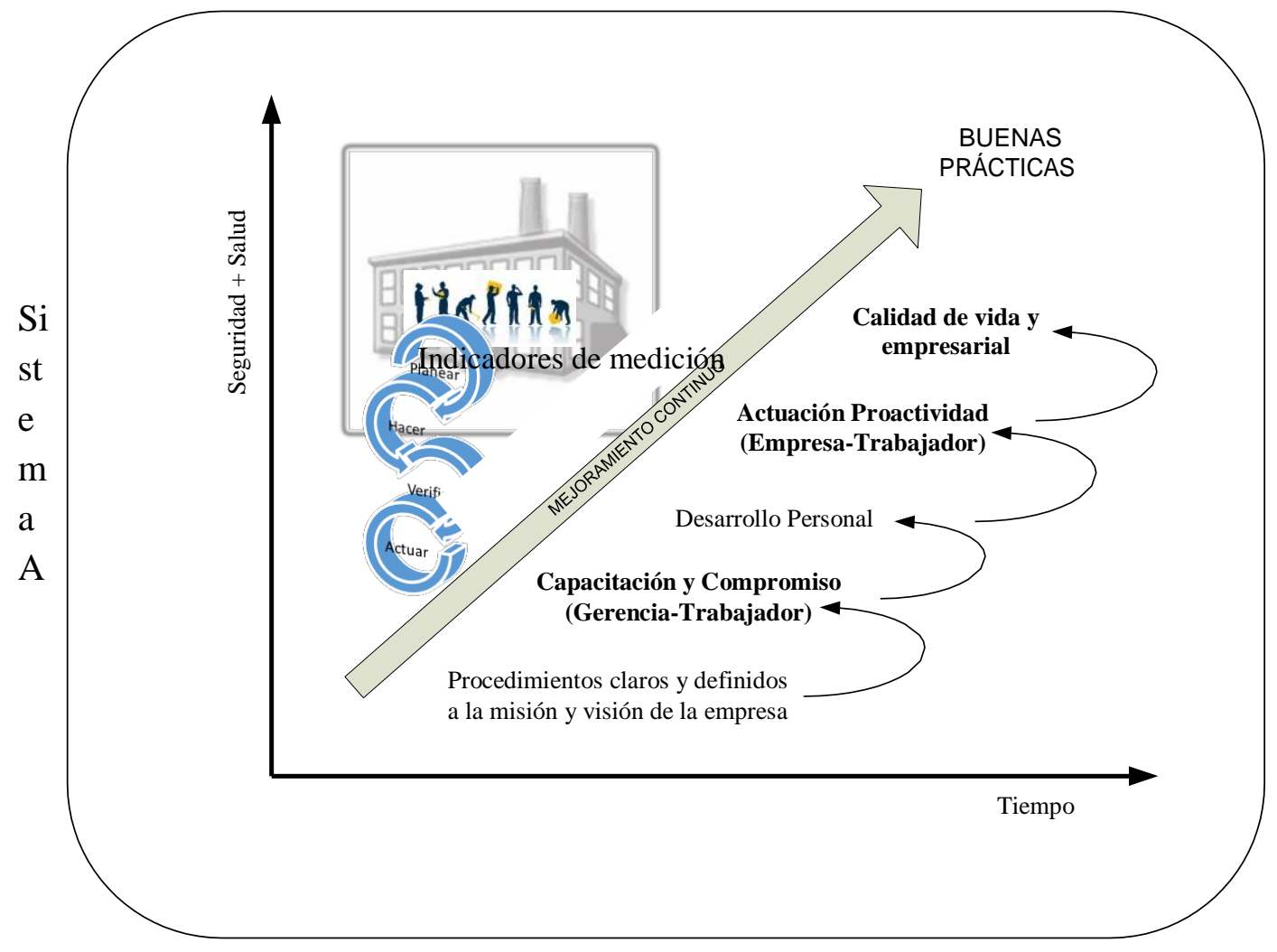

Fuente: Elaboración propia

Todo proceso es un sistema abierto donde intervienen multiples factores que permiten la ejecución del mismo.

La seguridad y salud del trabajo no debe trabajarse aisladamente, es decir, no solo la empresa es la encargada de hacer y ejecutar normas porque son exigidas gubernamentalmente o porque pueden beneficiar competitivamente a la misma. El trabajador quien hace vida y permite la operacionalización de los procesos también debe ser involucrado desde que inicia.

Se debe recordar que todos los que laboran en una empresa son personas y que por ello todos tienen el compromiso y responsabilidad por su seguridad y salud. 
La gestión de seguridad y salud del trabajo es una parte del sistema general de la gestión organización. La diferencia radica en que la primera se enfoca a la política de la prevención y la segunda a la misión y visión de la empresa. Sin embargo ambas deben trabajar en cadena, así como todos los procesos que se desarrollan en la empresa.

ISOTools, 2016 expresa que un sistema de gestión de seguridad y salud del trabajo es una disciplina que trata de prevenir las lesiones y enfermedades provocadas por las condiciones expuestas en la que se encuentra el trabajo, además de la protección y promoción de la salud. También expresa que todo sistema de gestión debe partir del principio de Deming el cual esta enfocado a PHVA (planificar, hacer, verificar y actuar).

Por lo expuesto anteriormente es que el modelo de gestión presentado en la figura 1, tiene representado la relación empresa-trabajador donde se integran hacia un fin común como es el manejo, y crecimiento de todos los procesos aplicando un mejoramiento continuo conducente a la incorporación de buenas prácticas. También considera la aplicación de medidas de control por medio del uso de indicadores de manera que se pueda medir y mejorar sus resultados o avances en el tiempo.

Por otro lado, el modelo de gestión propuesto parte de que además de considerar el ciclo de PHVA, también debe partir de procedimientos claros y definidos, capacitación y adiestramiento continuo de todo el personal que haga vida en la empresa,de manera que el mismo pueda responder de forma proactiva y no reactiva. Repercutiendo hacia la Calidad Total de los Procesos y del Ser Humano. 
La vida y salud estan primero. Pareciera ser que el trabajador no pensara las consecuencias por su accion conductual en función del contexto social donde se desenvuelve y de la posibilidad de alguna incapacitación física.

La seguridad es un compromiso de todos, por lo cual con conciencia y responsabilidad se puede lograr minimizar las enfermedades y las posibles lesiones que pudieran darse en el medio ambiente de trabajo.

\section{Conclusiones}

- Se evidencio un crecimiento exponencial desde el año 2010 al 2016 de accidentes laborales, $250 \%$ aproximadamente.

- Se generan ajustes nacionales e internacionales en los sistemas de gestión de salud del trabajo con el propósito de minimizar los riesgos y mejorar las condiciones del medio ambiente de trabajo.

- Las medidas de seguridad no solo se deben llevar desde un manual, también se debe gestionar a fin de integrar y comprometer a todo el personal que labora en la empresa.

- La cognición del trabajador presenta debilidades en la aplicación y ejecución de las normas de seguridad y de la salud laboral.

- La aplicación conjunta de las estrategias permitirán fortalecer la gestión de seguridad y salud del trabajo, porque presenta acciones que van más alla de un mejoramiento continuo.

- Todo sistema de gestión debe trabajar de forma integral (empresa-trabajador) para lograr fortalecer sus debilidades, y así poder aprovechar las oportunidades que se les pueda presentar.

- La seguridad es un compromiso de todos, por lo cual se le debe dar la importancia a fin de lograr resultados conducentes hacia la calidad. 


\section{Bibliografía.}

ALTAÏR. (07 de Junio de 2017). Sistema de Gestión en Seguridad y Salud Ocupacional. Recuperado el 05 de Agosto de 2017, de http://altairdelecuador.com/blog/2017/06/sistemagestion-seguridad-salud-ocupacional/

Chinchilla Sibaja, R. (2002). Salud y Seguridad en el Trabajo. Costa Rica: EUNED Universidad Estatal a Distancia.

Constitución de la República del Ecuador. (20 de Octubre de 2008). Obtenido de http://www.asambleanacional.gov.ec

El Telégrafo. (2012). El Telégrafo. Recuperado el 22 de Agosto de 2017, de http://tinyurl.com/zn4qg95

Fernandez, D. (2003). Conceptos de administración estratégica (Novena ed.). México: Pearson.

Gasoriowski-Denis, E. (12 de Febrero de 2016). ISO Organización Organizacional de Normalización. Recuperado el 01 de Agosto de 2017, de https://www.iso.org/2016/02/Ref2012.html

González Muñiz, R. (2003). Manual Básico Prevención De Riesgos Laborales. España: THOMSON.

Hernández, M. T. (2006). Manual de Trabajos de Grado de Especialización y Maestría y Tesis Doctorales (4a. ed.). Caracas, Venezuela: FEDUPEL.

Hurtado de Barrera, J. (2010). El proyecto de investigación (Sexta ed.). Caracas: Sypal.

ISO. (s.f.). Organización Internacional de Normalización. Recuperado el 17 de agosto de 2017, de https://www.iso.org/iso-45001-occupational-health-and-safety.html

ISOTools. (16 de Septiembre de 2016). Blog Calidad y Excelencia. 
Organización Internacional del Trabajo. (2014). OIT Organización Internacional del Trabajo. Recuperado el 20 de Agosto de 2017, de http://www.ilo.org/global/about-theilo/newsroom/news/WCMS_301241/lang--es/index.htm

Sotomayor, D. (29 de Septiembre de 2016). Expreso.ec. Recuperado el 24 de Agosto de 2017, de http://www.expreso.ec/guayaquil/accidenteslaborales-estres-capacitacionesrecomendaciones-estadisticas-IY724587 